\title{
Epidemiologia dos pacientes hipertensos de uma estratégia saúde da família de Belém - Pará
}

\author{
Epidemiology of hypertensive patient in a family health strategy in Belém - Pará \\ Epidemiología de pacientes hipertensos en una estratégia salud familiar en Belém - Pará \\ Juliana Joyce Chaves de Lima ${ }^{1 *}$, Ivan Dias Neto ${ }^{1}$, Israela Cristine Pereira Marinho ${ }^{1}$, Ana Karina \\ Corôa Vasconcelos ${ }^{1}$, Natália Tavares Carvalho ${ }^{1}$, Elton Cardoso Pinheiro ${ }^{1}$, Jeanne Seabra Negrão \\ Lima da Silva1, Rafaella Pereira Marinho ${ }^{1}$, Lorena Batista de Lemos Ghammachi ${ }^{1}$, Deusa Meriam \\ da Silva Brito'.
}

\section{RESUMO}

Objetivo: Descrever a evolução da prevalência da hipertensão arterial na comunidade nos anos de 2010 a 2014 e o perfil dos pacientes cadastrados. Métodos: O estudo foi do tipo retrospectivo, quantitativo, observacional e descritivo, realizado em uma Unidade de Saúde da Família através da análise de 755 prontuários dos pacientes hipertensos cadastrados. O projeto foi aprovado pelo Comitê de Ética em Pesquisa (CEP) sob CAAE 51481115.2.0000.5701. Resultados: Os resultados demonstraram que a prevalência aumentou no decorrer dos anos conforme o esperado, tendo aumentado principalmente no ano de 2014, fechando o período com o índice de 15,80\%. Constatou-se também que a Hipertensão Arterial Sistêmica (HAS) é a mais prevalente em pacientes com renda de até 3 salários mínimos, baixa escolaridade e idosos até 70 anos. Conclusão: Essas variáveis tiveram grande representatividade quando associados a prevalência de hipertensão, visto que esses fatores influenciam diretamente no processo de prevenção e promoção da saúde.

Palavras-chave: Hipertensão, Atenção primária à saúde, Epidemiologia.

\begin{abstract}
Objective: To describe the evolution of the prevalence of arterial hypertension in the community from 2010 to 2014 and the profile of registered patients. Methods: The study was retrospective, quantitative, observational and descriptive, conducted at the Family Health Unit of through the analysis of 755 medical records of registered hypertensive patients. The project was approved by the Research Ethics Committee under CAAE 51481115.2.0000.5701. Results: The results showed that the prevalence increased over the years as expected, having increased mainly in 2014 , closing the period with the rate of $15.80 \%$. It was also found that Systemic Arterial Hypertension (SAH) is more prevalent in patients with an income of up to 3 minimum wages, low education and elderly up to 70 years. Conclusion: These variables were highly representative when associated with the prevalence of hypertension, since these factors directly influence the process of prevention and health promotion.
\end{abstract}

Keywords: Hypertension, Primary health care, Epidemiology.

\section{RESUMEN}

Objetivo: Describir la evolución de la prevalencia de hipertensión arterial en la comunidad de 2010 a 2014 y el perfil de pacientes registrados. Métodos: El estudio fue retrospectivo, cuantitativo, observacional y

${ }^{1}$ Centro Universitário Metropolitano da Amazônia (UNIFAMAZ), Belém - Pará

*E-mail: juliana.chaves@msn.com 
descriptivo, realizado en la Unidad de Salud de la Familia a través del análisis de 755 registros médicos de pacientes hipertensos registrados. El proyecto fue aprobado por el Comité de Ética de Investigación bajo CAAE 51481115.2.0000.5701. Resultados: Los resultados mostraron que la prevalencia aumentó a lo largo de los años como se esperaba, habiendo aumentado principalmente en 2014, cerrando el período con una tasa del 15,80\%. También se descubrió que la Hipertensión Arterial Sistémica (HSA) es más frecuente en pacientes con ingresos de hasta 3 salarios mínimos, baja educación y ancianos de hasta 70 años. Conclusión: Estas variables fueron altamente representativas cuando se asociaron con la prevalencia de hipertensión, ya que estos factores influyen directamente en el proceso de prevención y promoción de la salud.

Palabras clave: Hipertensión, Atención primaria de salud, Epidemiología.

\section{INTRODUÇÃO}

A hipertensão é caracterizada por níveis elevados de pressão sanguínea nas artérias, é uma das maiores causas de morbidade e mortalidade no mundo, considerada como um grave problema de saúde pública brasileira, sendo responsável pelos altos custos com internações, incapacitação por invalidez e antecipação da aposentadoria, atingindo todas as classes socioeconômicas no Brasil (SOUSA JCL e LOPES LS, 2014).

A Organização Mundial da Saúde (OMS) considera HA a partir da medida aferida acima de $140 \mathrm{mmHg}$ e/ou pressão diastólica igual ou superior a $90 \mathrm{mmHg} 1,16$, enquanto outros estudos consideram a medida aferida igual ou acima de $140 \mathrm{mmHg} / 90 \mathrm{mmHg}$, ou mediante o uso atual de medicação anti-hipertensiva (MALTA DC, et al., 2018).

No Brasil, até $25 \%$ da população adulta possui essa patologia e é estimado que em 2025, esse número cresça para $60 \%$, atingindo uma prevalência geral de $40 \%$. A Hipertensão Arterial Sistêmica (HAS), além de acarretar custo social e econômico elevado com uma vida produtiva parada, é uma das principais causas de mortes por problemas de sistema circulatório (MOREIRA JPL, et al., 2013).

Segundo a Sociedade Brasileira de Hipertensão, o país possui atualmente 17 milhões de pessoas que sofrem de pressão alta, sendo 420 mil óbitos anuais em consequência de Acidente Vascular Cerebral (AVC) e 1,2 milhão de óbitos em decorrência de males cardiovasculares relacionados à doença, podendo ainda, afetar alguns órgãos como coração, cérebro, rins e vasos sanguíneos (ZATTAR LC, et al., 2013).

A Hipertensão arterial apresenta uma evolução lenta e silenciosa. A alteração é geralmente diagnosticada de forma tardia e, na maioria das vezes, não é possível identificar com exatidão a causa do aumento da pressão arterial no paciente, no entanto, é possível associa-la a alguns fatores naturais como hereditariedade, idade avançada e o sexo, e a fatores externos, como a falta de atividade física que acarreta outro fator importante de risco: o excesso de peso (BORGES H, et al., 2008). A má alimentação com uso excessivo de sal pode facilitar ou agravar a hipertensão assim como o álcool, tabagismo e estresse, sendo os três últimos os fatores de risco mais comuns (ZATTAR LC, et al., 2013).

Apesar disso, realizar atividade física regularmente, alimentação com controle e pouca quantidade de sal além de tomar as medicações recomendadas pelo profissional médico que controla a pressão arterial alta, são indicações do tratamento desta doença que, quando controlado, possui um prognóstico favorável ao paciente (MOREIRA JPL, et al., 2013).

Estudos com medidas aferidas da Pressão Arterial (PA) são escassos na população brasileira, sendo os estudos, na sua maioria, locais e com grande variabilidade de informações, o que inviabiliza a comparação de dados. Além disso, existem diferentes critérios diagnósticos para estimar a prevalência populacional de HA (ZATTAR LC, et al., 2013).

A baixa adesão ao tratamento encontrada frequentemente nos níveis de atenção primária à saúde dificulta o controle da pressão arterial e consequentemente, a diminuição de lesões encontradas nos órgãos afetados (LIMA T, et al., 2010). O abandono do regime terapêutico também é comum, decorrência da dificuldade em 
executar mudanças no estilo de vida, incluindo a prática de atividades físicas regularmente, e a adoção de uma dieta alimentar hipossódica, além de que, muitos pacientes esquecem a medicação diariamente. A escassez de informação também influencia no aumento de hipertensos e contribui para as complicações, razão pela qual, o trabalho dos agentes de saúde das Unidades de Atenção Básica possui grande importância', além de possui contado diário com o paciente orientando e diminuindo o risco do paciente esquecer de tomar a medicação ou de ir à consultas marcadas (LIMA T, et al., 2010).

Considerando-se tais afirmativas, a pesquisa estudou a prevalência da hipertensão arterial, haja visto que para o desenvolvimento de estratégias novas que possibilitem a adesão maior ao tratamento, será necessário, conhecer a evolução da doença na área, assim como o perfil dos pacientes cadastrados na área de uma Unidade de Saúde da Família do estado do Pará. O objetivo da pesquisa foi descrever a evolução da prevalência da hipertensão arterial (HAS) em uma comunidade nos anos de 2010 a 2014 e o perfil dos pacientes cadastrados na Unidade de Saúde da Família da localidade.

\section{MÉTODOS}

O presente estudo foi realizado segundo os preceitos da Declaração de Helsinque e do Código de Nuremberg, respeitando as normas de pesquisas envolvendo seres humanos (Res. CNS 466/12) do Conselho Nacional de Saúde, aprovado pelo Comitê de Ética em Pesquisa sob CAAE 51481115.2.0000.5701, pela instituição responsável pelos dados e autorização dos pacientes ou responsáveis, por meio de termo de consentimento livre e esclarecido.

O estudo foi do tipo retrospectivo, quantitativo, observacional e descritivo, realizado em uma Unidade de Saúde da Família no estado do Pará, abrangendo os anos de 2010 a 2014. A coleta de dados ocorreu após a aprovação do comitê de ética em pesquisa. Para a obtenção da amostra foram verificados os registros das equipes I e II da Unidade obtendo-se uma amostra de 755 hipertensos, número de cadastramentos até o ano de 2014 da Unidade.

Os 755 hipertensos que constituíram a casuística do estudo, foram estudados de acordo com as micro áreas cobertas pela Unidade. A equipe I responsável pela micro área I da comunidade possuía 404 hipertensos e a equipe II responsável pela micro área II possuía 351 hipertensos.

Para coleta de dados, foi utilizado protocolo de pesquisa elaborado pelos pesquisadores, onde cada pesquisador coletou informações de micro áreas diferentes, analisando em cada prontuário as variáveis referentes à idade, sexo, cor, renda, peso e adesão ao tratamento.

Para avaliar a regularidade no tratamento, variável considerada como base para avaliar a adesão ao tratamento, considerou-se em tratamento regular, os pacientes com diagnóstico de hipertensão que comparecem a $80 \%$ das consultas agendadas para acompanhamento durante 0 ano. De outro modo, 0 tratamento foi considerado irregular.

No que concerne ao cálculo de Índice de Massa Corporal (IMC), não foi possível realizar a coleta de dados na amostra, decorrente da falta de preenchimento dos dados de peso e altura nos prontuários. $\mathrm{O}$ cálculo de IMC seria importante visto que grande parte dos pacientes hipertensos possuem IMC alterado e a HAS está associada à obesidade e hábitos de vida saudáveis.

As principais limitações do estudo das informações coletadas foram devido ao preenchimento incorreto dos prontuários, além da falta de atualização, ausência dos pacientes as consultas marcadas, ilegibilidade das letras de alguns profissionais e falta de prontuários nos arquivos. Os resultados obtidos na coleta foram tabulados por meio do programa Microsoft Office Excel (2010) e Microsoft Office Word (2010) sendo construídos gráficos demonstrativos dos resultados.

Foram incluídos $100 \%$ dos pacientes cadastrados como hipertensos na Unidade de Saúde no Sistema HIPERDIA (Hipertensão - Diabetes), independentemente de sexo, idade, raça, cor, religião ou da existência de outras patologias associadas. Foram excluídos da pesquisa os pacientes que recusaram a participação, assim como aqueles que se encontrava em tratamento, mas, por algum motivo, não foram cadastrados no Sistema HIPERDIA. 
A pesquisa trouxe como benefício principal a disponibilização de dados que podem ser úteis para o planejamento das ações da Unidade, e poderão servir também como modelo para outros estudos no município. Além disso, os pacientes foram beneficiados com ações efetivas de informação sobre o agravo, e tiveram o melhor cuidado por parte das equipes de acompanhamento.

Os pesquisadores também foram beneficiados no seu aprendizado sobre este agravo de saúde, importante fato na sua formação acadêmica. Quanto aos riscos previstos para os pacientes, a quebra de sigilo de seus dados, foi evitada pelo exclusivo manuseio pelos pesquisadores, com a guarda adequada do material pelo tempo determinado pelas normas com a destruição sigilosa dos mesmos após o tempo do encerramento desse estudo.

Os riscos para os pesquisadores e para a comunidade científica foi decorrente de informações incorretas coletadas que poderia resultar em análises errôneas, o que exigiu grande cuidado na análise dos dados coletados.

\section{RESULTADOS}

Por meio da coleta de dados da pesquisa, foram encontrados informações sobre diversas variáveis de características pessoas da população estudada. Em relação a cor/raça, 123 pacientes não possuíam esta informação nos prontuários (16\%), 525 eram pardos (70\%), 63 negros (8\%) e 44 brancos $(6 \%)$.

Já quanto ao número de pacientes hipertensos cadastrados na Unidade de saúde estudada distribuídos por faixa etária, 123 não possuíam informação de idade, 23 tinham menos que 40 anos, 59 tinham entre $40 \mathrm{e}$ 49 anos, 167 tinham entre 50 e 59 anos, 193 tinham entre 60 e 69 anos, 108 tinham entre 70 e 79 anos e 82 tinham mais que 80 anos de idade.

Foi distribuído também, uma divisão por sexo dos prontuários de pacientes hipertensos, sendo que 123 não possuíam a informação de sexo (16\%), 395 eram do sexo feminino (52\%) e 237 eram do masculino (32\%)

A partir da análise de características socioeconômicas da população estudada, foram encontradas as seguintes informações. Analisando a renda mensal, 123 não possuíam esta informação no prontuário, 45 possuíam menos que 1 salário mínimo, 549 possuíam entre 1 e 3 salários mínimos, 38 possuíam mais que 3 salários mínimos (Gráfico 1).

Gráfico 1 - Número de pacientes hipertensos cadastrados da Unidade de Saúde distribuídos de acordo com a renda mensal.

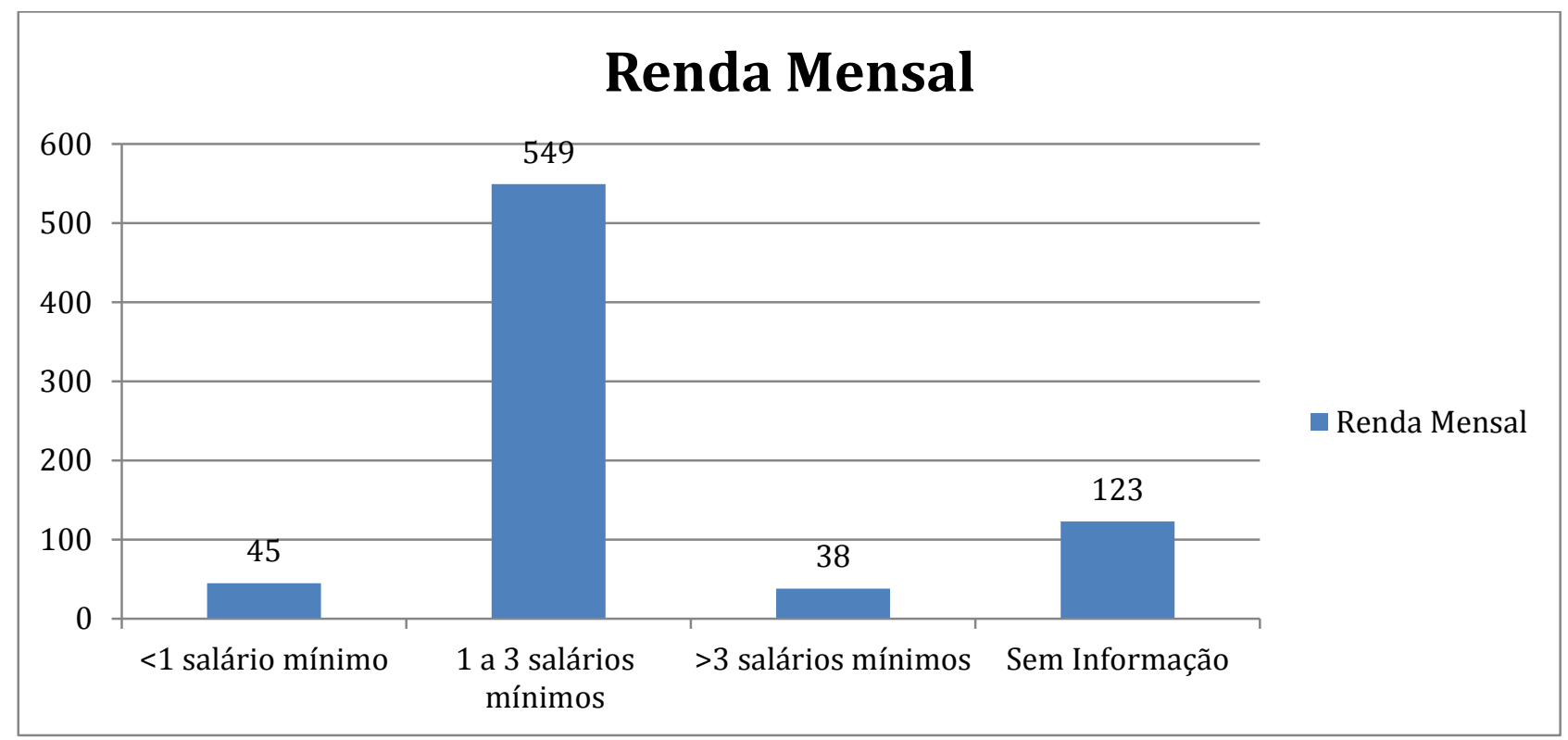

Fonte: Lima JJC, et al., 2020. 
Analisando o grau de escolaridade da população estudada, 123 não possuíam tal informação no prontuário, 31 eram analfabetos, 303 eram alfabetizados, 164 possuíam fundamental incompleto, 57 com fundamental completo, 49 possuíam ensino médio incompleto, 28 possuíam médio completo, 0 ensino superior completo ou incompleto (Gráfico 2).

Gráfico 2 - Número de pacientes hipertensos cadastrados da Unidade de Saúde distribuídos de acordo com seu grau de escolaridade.

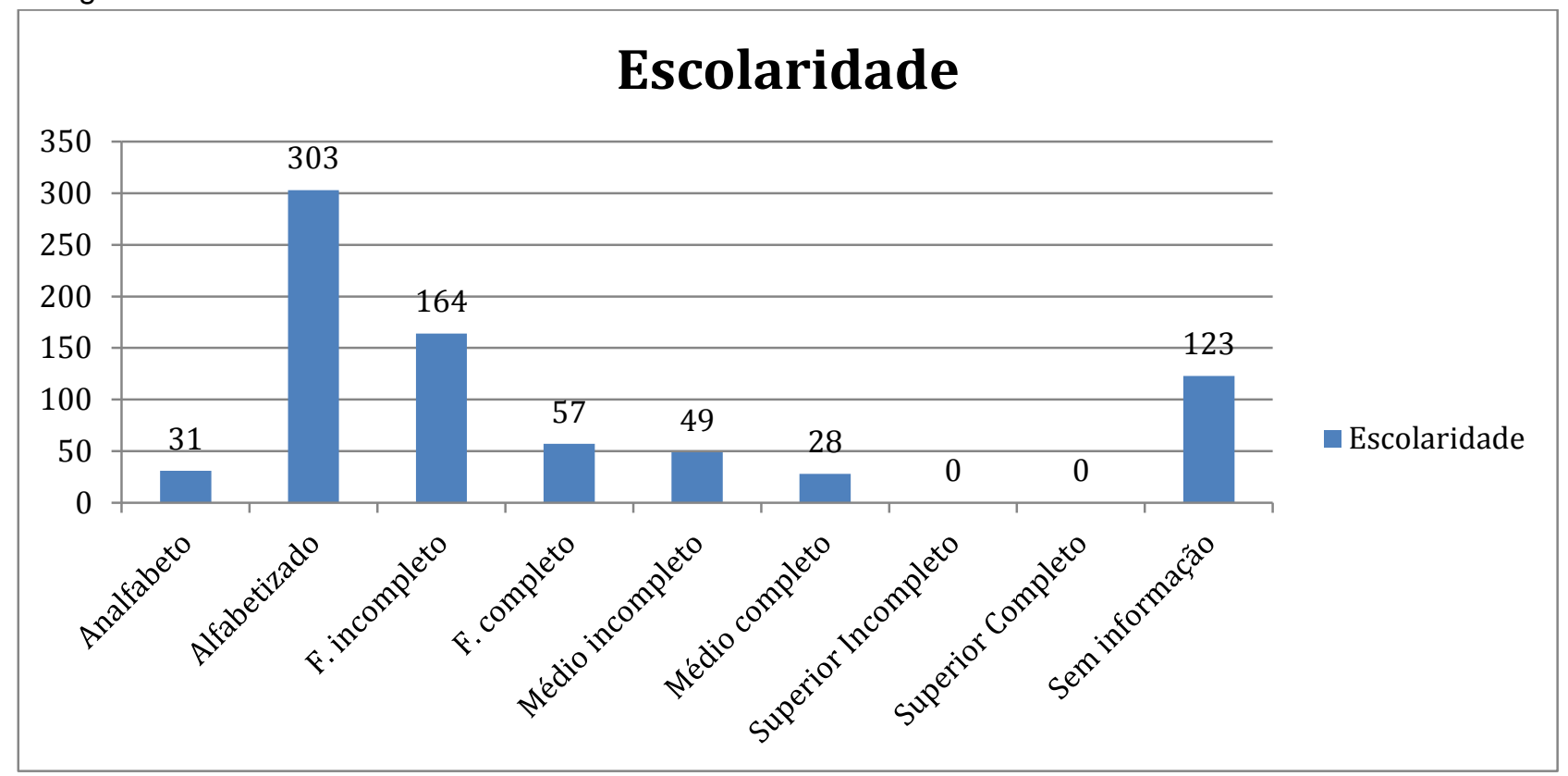

Fonte: Lima JJC, et al., 2020.

Foi analisado características do tratamento da população estudada. Do universo de pacientes, 123 não continham informação de quando iniciou a adesão ao tratamento da Hipertensão Arterial Sistêmica, 251 iniciaram antes de 2010, 73 em 2010, 78 em 211, 69 em 2012, 83 em 2013 e 78 em 2014 (Gráfico 3).

Gráfico 3 - Número de pacientes hipertensos cadastrados da Unidade de Saúde distribuídos de acordo com seu ano de adesão ao tratamento.

\section{Ano de adesão}

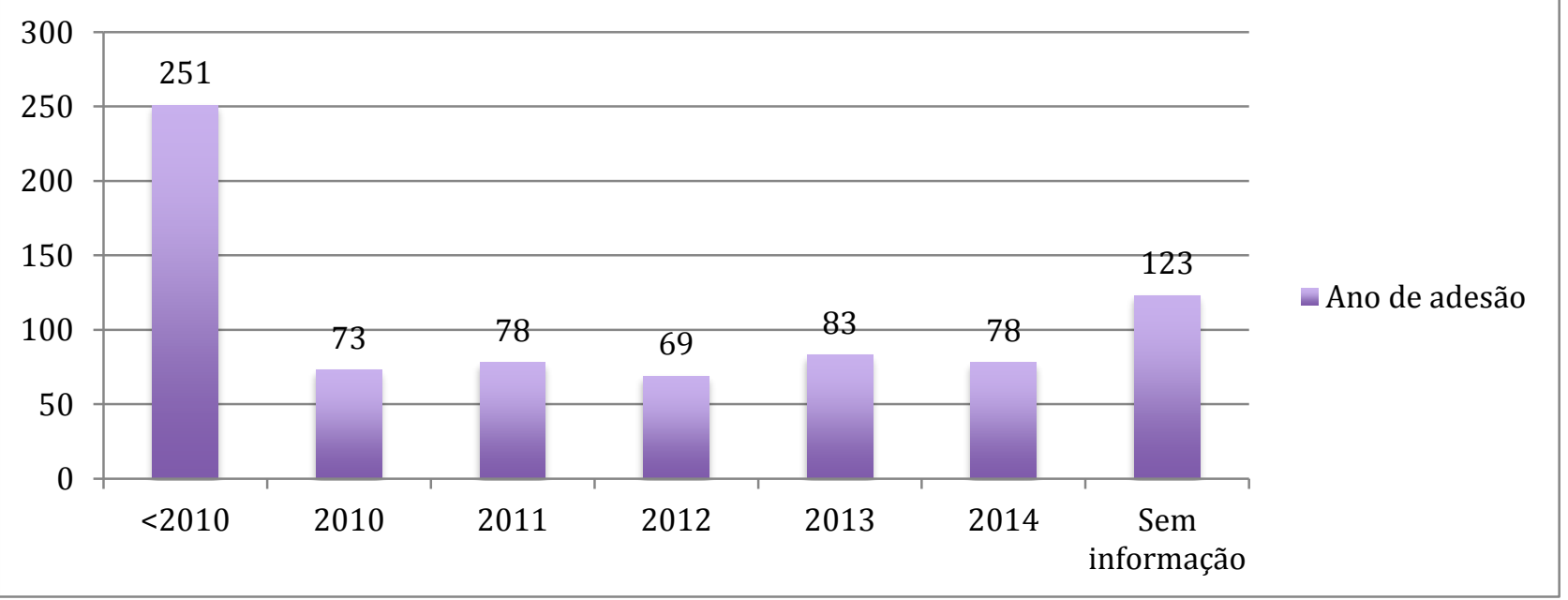

Fonte: Lima JJC, et al., 2020. 
Já analisando os casos acumulados de hipertensão arterial sistêmica cadastrados na unidade estudada, 123 não continham estas informações, 251 foram cadastrados antes de 2010, 324 em 2010, 402 em 2011, 471 em 2012, 554 em 2013 e 632 em 2014 (Gráfico 4).

Gráfico 4 - Casos acumulados de hipertensão arterial sistêmica cadastrados da Unidade de Saúde.

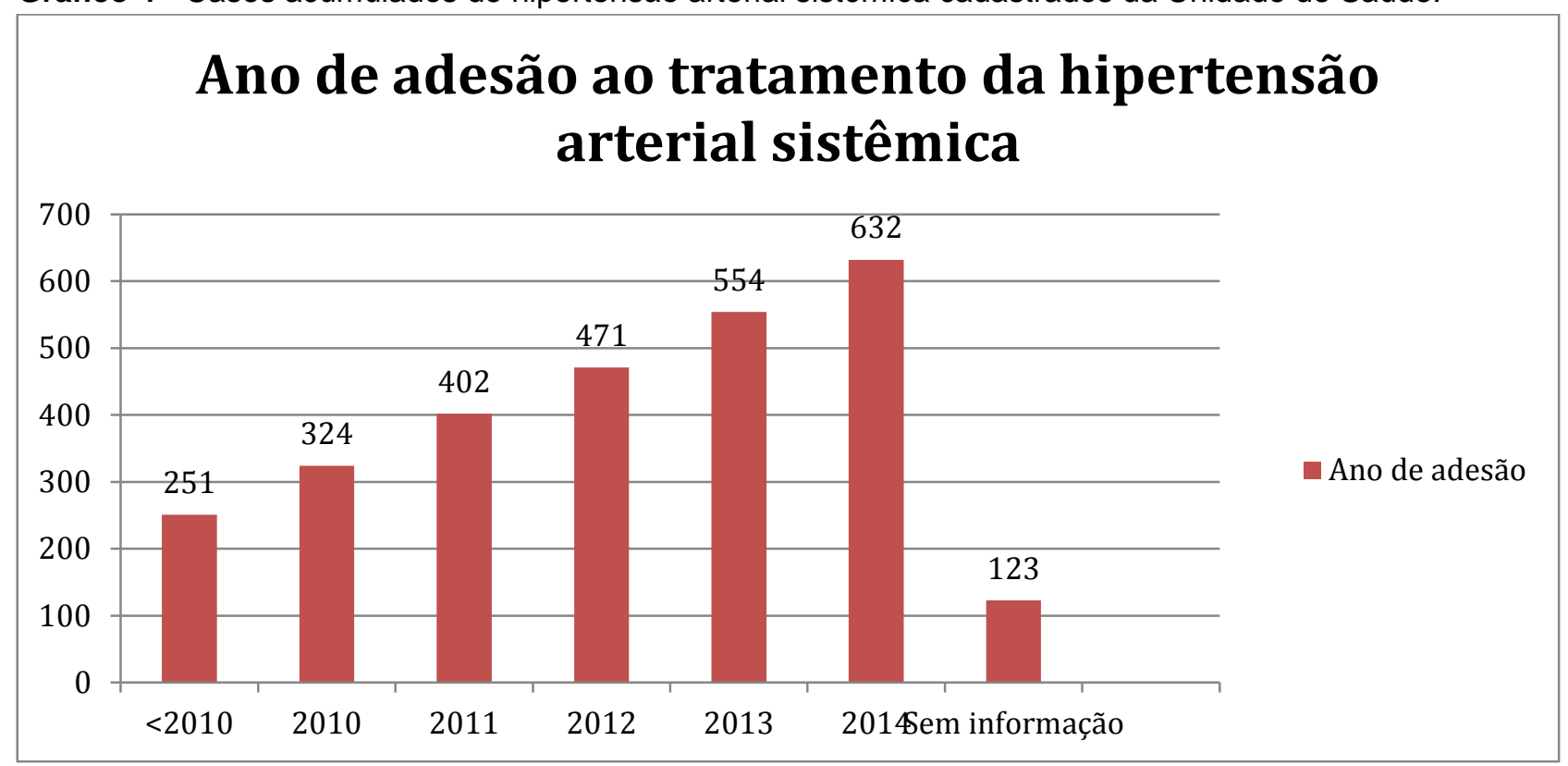

Fonte: Lima JJC, et al., 2020.

Por último, analisando a prevalência de hipertensão na unidade no período estudado, foi encontrado uma elevação sendo 6,27\% antes de 2010, 8,10\% em 2010, 10,05\% em 2011, 11,77\% em 2012, 13,85\% em 2013 e 15,80\% em 2014 (Gráfico 5).

Gráfico 5 - Prevalência da hipertensão no período de 2010 a 2014 na Unidade de Saúde.

\section{Prevalência anual}

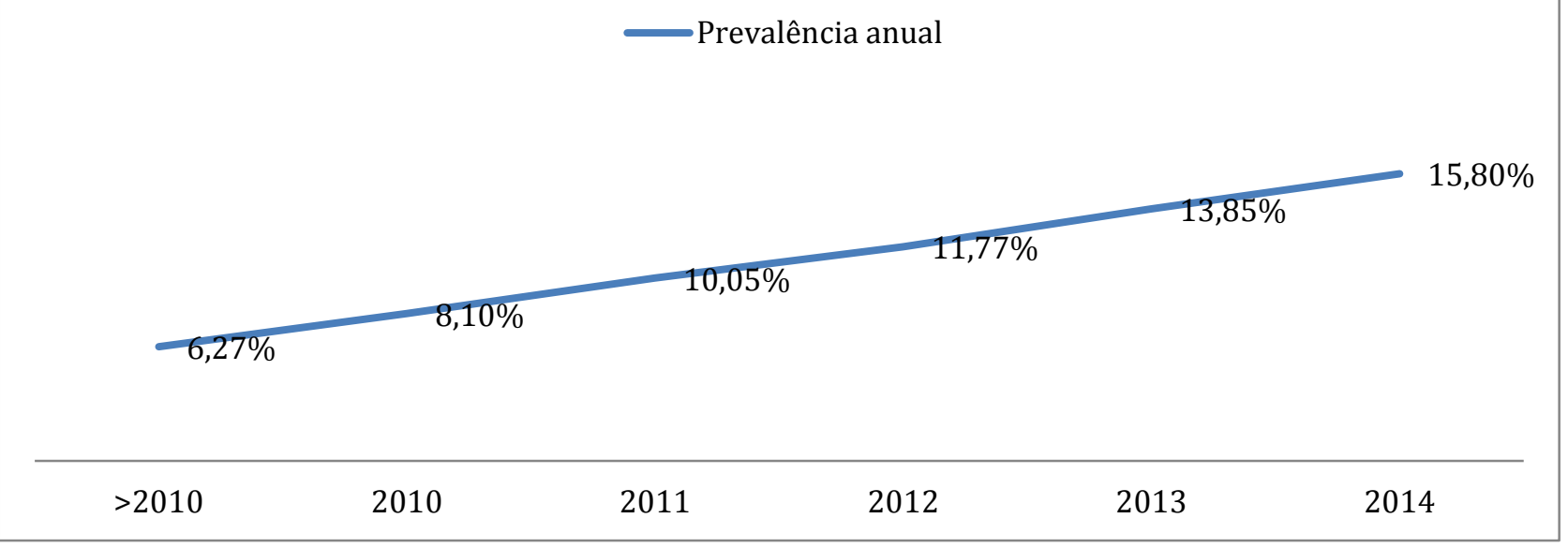

Fonte: Lima JJC, et al., 2020.

Quanto às informações de número de pacientes hipertensos acompanhados conforme regularidade, 123 não tinham esta informação (16\%), 512 eram acompanhados de forma irregular (68\%) e 120 de forma regular (16\%) (Gráfico 6). 
Gráfico 6 - Número de pacientes hipertensos acompanhados na Unidade de Saúde conforme a regularidade do tratamento.

\section{Tratamento}

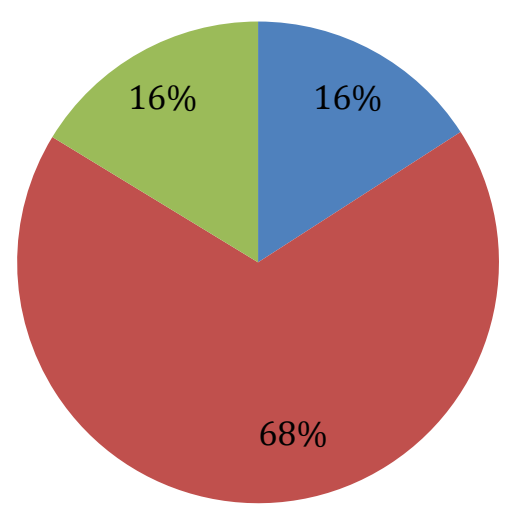

- Regular

- Irregular

- Sem informação

Fonte: Lima JJC, et al., 2020.

\section{DISCUSSÃO}

A HAS é a doença crônicas mais prevalente na sociedade brasileira, principalmente em idosos após 60 anos de idade. Em dados da Pesquisa Nacional de Saúde (2014), mostram que a HAS atingiu prevalência de 21,40\% em pessoas acima de 18 anos (MENDES G, et al., 2014).

No presente estudo, observou-se que a predominância da HAS foi maior em indivíduos do sexo feminino, sendo encontrados $52 \%$ em relação aos $32 \%$ do sexo masculino, excluindo $16 \%$ dos prontuários médicos por não constarem dados suficientes para a pesquisa, conforme mostrado na seção de resultados. Esta prevalência encontrada aproxima-se da pesquisa de Converso (2015) que foi superior a $80 \%$ no sexo feminino, haja vista que mulheres procuram mais serviços de saúde facilitando o diagnóstico de HAS (MENDES G, et al., 2014).

A literatura analisa que alguns dados de prevalência e epidemiologia da HAS entre os sexos existem. Em mulheres, por exemplo, o nível da pressão arterial média é influenciado por algumas situações e contextos, tais como doenças (síndrome do ovário policístico - SOP), reposição hormonal e menopausa após os 50 anos de idade, o uso de anticoncepcional (seja oral, seja intradérmico ou por injeção), período gestacional, podendo levar em algumas dessas situações ao aumento da pressão arterial média e por fim ao desenvolvimento da HAS (COLOMBO FC, 2011; SOUZA NP, et al., 2020).

Alguns outros contextos e situações na vida da mulher também podem justificar esse aumento da pressão arterial média, como a entrada no meio profissional e de trabalho. O nível de estresse feminino vem aumentando nos últimos anos pela maior participação e entrada das mulheres no mercado de trabalho que fazem se sobrecarregar haja vista muitas, ao chegar do trabalho ainda possuem educação dos filhos, contas à pagar e ambiente doméstico para resolver sem ter auxílio de parceiro, podendo levar ao aumento da sobrecarga das exigências solicitadas pelo ambiente profissional que, juntas com atribuições domésticas sem apoio de outra pessoa, acaba por levar à níveis mais elevados de estresse nas mulheres, sendo fator precipitante e importante para o desenvolvimento de Hipertensão Arterial Sistêmica (NOBLAT ACB, et al., 2014).

Analisando a variável cor da presente pesquisa, observou-se a que na população estudada, houve a predominância de $70 \%$ de cor parda, porém, não sendo considerado significativo por ser a cor predominante $\mathrm{e}$ isso deve ser levado em consideração pois, geneticamente, as pessoas da raça negra possuem mais probabilidade de vir a desenvolver a hipertensão arterial sistêmica (NOBLAT ACB, et al., 2014). 
Neste sentido, as pessoas de raça negra, por terem mais predisposição à Hipertensão Arterial Sistêmica deveriam tomar mais medidas de cuidado e prevenção desta doença tais como atividade física regular, alimentação saudável e nutritiva além da avaliação periódica ao profissional médico para rastreio desta patologia. (LIMA NKC, et al., 2020).

No que se refere à idade, nos prontuários examinados a hipertensão arterial sistêmica foi predominante em $26 \%$ dos idosos na faixa etária de 60 a 69 anos, demonstrados na parte de resultados. Observamos uma associação direta entre a prevalência da doença e a idade, em conformidade com outros estudos, já que em nossa pesquisa a prevalência também ocorreu em idosos, haja vista as artérias dos idosos estão com pouca quantidade de tecido muscular e por isso estão menos propensas a variações de fluxo sanguíneo, dessa forma mantendo a hipertensão arterial sistêmica mais constante (COLOMBO FC, 2011; NOBLAT ACB, et al., 2014; YUGAR-TOLEDO JC, et al., 2020).

Sendo este fator idade importante para a saúde pública, haja vista que a doença Hipertensão Arterial Sistêmica estar associada ao aumento de idade, os idosos deveriam melhorar os métodos de prevenção (atividade física, alimentação saudável e visitas periódicas ao médico para rastreio) ou tratar de forma adequada (não esquecer de utilizar a medicação e não utilizar de forma errônea) para que não ocorra desenvolvimento de doenças e consequências sérias como o Acidente Vascular Encefálico (AVE) ou Infarto Agudo do Miocárdio (IAM) gerando prejuízo à vida biopsicossocial do paciente (BRANDAO AA, et al., 2010).

Em relação às características socioeconômicas, no Gráfico 1 podemos verificar que a renda foi um fator determinante, visto que a prevalência ocorreu em 73\% dos pacientes que possuíam de 1 a 3 salários mínimos, aproximando-se do estudo de Radovanovic CAT (2014), que informa que a classe econômica mais prevalente também seria a que possuía renda de um a três salários mínimos, com $31,82 \%$ de indivíduos entre os hipertensos. A renda possui fator determinante pois, há indícios que quanto menos renda familiar, menos diversificada e com mais quantidade de sal nos alimentos as famílias ingerem, aumento a possibilidade da doença (COLOMBO FC, 2011; PASSOS VMS, et al., 2016).

O nível de escolaridade (Gráfico 2), também influenciou e é um dos mais importantes indicadores de condições de saúde da população, além de estar relacionado à idade já que boa parte da população idosa não concluiu ou iniciou seus estudos. Este estudo demonstrou que quanto menor o nível de escolaridade, maior a prevalência da hipertensão arterial sistêmica, uma vez que $40 \%$ da população estudada eram apenas alfabetizada, seguido de $22 \% \mathrm{com}$ ensino fundamental incompleto, $8 \%$ correspondente ao ensino fundamental completo, $6 \%$ ao ensino médio incompleto, $4 \%$ ao ensino médio completo, $4 \%$ de analfabetos e $0 \%$ relacionado ao nível superior completo e nível superior incompleto, com $16 \%$ equivalentes a pacientes com prontuários sem informações suficientes. Estes dados aproximam-se do estudo realizado por Oliveira BFA (2013), o qual, afirma que em indivíduos com menores de 2 anos de estudos, a hipertensão tornava-se prevalente, além do estudo de Araújo JC (2006) que 94,2\% tinham no máximo, o primeiro grau completo, haja vista encontrarem explicação que quanto menor o grau de estudo mais provável do indivíduo se alimentar de maneira errada e não realizar exercícios de maneira eficiente e regular, aumentando a probabilidade de vir a desenvolver a hipertensão arterial sistêmica.

Quanto ao tratamento (Gráfico 5), 68\% realizavam tratamento de maneira irregular, contrastando com o estudo de Araujo JC (2006) onde 91,8\% dos pacientes analisados apresentaram tratamento regular. Esse fato evidencia a necessidade de ações voltadas para a prevenção da HAS nesta comunidade, além de confirmar a relação com a renda e principalmente escolaridade, pois indivíduos com menor instrução têm acesso precário ao sistema de saúde, recebem menos informações e não compreendem a importância do problema e da adesão ao tratamento, além de não possuírem condições financeiras de adquirirem medicamentos, quando estes faltam nas unidades ou de possuírem uma alimentação saudável (ARAUJO JC, 2006; ANDRADE SSA et al., 2015).

No que diz respeito aos Gráfico 3 e 4, referentes aos anos estudados, em 2010 foram cadastrados 73 pacientes de ambos os sexos, totalizando neste ano 324 pacientes com hipertensão. No ano de 2011 foram realizados os cadastros de 78 hipertensos, totalizando 402 pacientes na Unidade de Saúde. No ano seguinte, foi realizado o cadastro de 69 hipertensos, resultando em 471 pacientes. No ano de 2013, ocorreu o cadastro 
de 83 hipertensos, totalizando 554 pacientes com hipertensão cadastrados na unidade de saúde e em 2014, foram cadastrados 78 hipertensos, totalizando 632 pacientes, sendo excluídos os 123 pacientes que não possuíam informações completas nos prontuários médicos, demonstrando um aumento da quantidade de pacientes hipertensos cadastrados na unidade de saúde. Esse aumento no número de casos encontra apoio no diagnóstico mais eficiente dos pacientes com o passar dos anos, e com o aumento da longevidade dos moradores da área que desenvolveram a hipertensão arterial sistêmica por causas genéticas ou externas (falta de alimentação saudável e atividade física).

No que concerne ao Gráfico 6, nota-se um aumento da prevalência de hipertensão na comunidade conforme o esperado, encerrando o período com o índice de 15,80\%. No entanto, comparados aos dados da pesquisa Nacional de Saúde do ano de 2014, esse índice foi considerado baixo, o que pode evidenciar falha no diagnóstico da doença na população, o que pode levar a diversas complicações como a subnotificação dessa doença, complicando a vida de vários grupos populacionais que aumentam seus riscos de desenvolvimento de outras comorbidades como Infarto Agudo do Miocárdio, Diabetes Mellitus e Acidente Vascular Encefálico (AVE).

\section{CONCLUSÃO}

Levando em consideração os dados coletados e analisados, em relação à hipertensão arterial sistêmica na comunidade, demonstrou-se que a prevalência aumentou no decorrer dos anos conforme o esperado, tendo aumentado principalmente no ano de 2014, fechando o período com o índice de 15,80\%. Porém, em relação aos dados da pesquisa Nacional de Saúde do ano de 2014, esse índice foi considerado baixo, o que pode evidenciar falha no diagnóstico da doença na população. Foi constatado também, que a maioria da população estudada era do sexo feminino, de cor parda, na faixa de 60 a 69 anos e tinha baixa escolaridade associada a uma condição socioeconômica precária e não faziam tratamento regular. A falta de dados nos prontuários dos pacientes, a ilegibilidade e a ausência de prontuários sugerem que outros estudos sobre esse tema com abordagem desses aspectos pelos pesquisadores sejam realizados.

\section{REFERÊNCIAS}

1. ANDRADE SSA, et al. Prevalência de hipertensão arterial autorreferida na população brasileira: análise da Pesquisa Nacional de Saúde, 2013. Epidemiol. Serv. Saúde, Brasília , 2015; 24(2): 297-304.

2. ARAÚJO JC, et al. Controle da hipertensão arterial em uma unidade de saúde da família. Control of arterial hypertension in a family care unit. Fundação Bahiana para o Desenvolvimento das Ciências. Escola Bahiana de Medicina e Saúde Pública. Salvador, BA, Brasil. 2006; 41(3): 368-374.

3. BORGES H, et al. Associação entre Hipertensão Arterial e Excesso de Peso em Adultos, Belém, Pará, 2005. Faculdade de Nutrição - Instituto de Ciências da Saúde - Universidade Federal do Pará (UFPA). 2008; 91 (2):110-118.

4. BRANDAO AA, et al. Conceituação, epidemiologia e prevenção primária. J. Bras. Nefrol., São Paulo, 2010; 32(supl. 1): 1-4.

5. COLOMBO FC. Hipertensão arterial na mulher. In: Paola AAV, Barbosa MM, Guimarães JI. Cardiologia: livro texto da Sociedade Brasileira de Cardiologia. São Paulo: Manole; 2011, 2(4): 628-30.

6. CONVERSO ME, et al. Prevalência da hipertensão arterial e analise de seus fatores de risco nos núcleos de terceira idade de Presidente Prudente. Revista Ciência em Extensão. 2005, 2(1):4-10.

7. LIMA T, et al. Perfil de adesão ao tratamento de pacientes hipertensos atendidos na Unidade Municipal de Saúde de Fátima, em Belém, Pará, Amazônia, Brasil. 2010, 1(2):113-120.

8. LIMA, Nereida Kilza da Costa. Redução de Sal na Dieta: Ilusão ou Realidade?. Arq. Bras. Cardiol., São Paulo, 2020, 114(3): 562-563.

9. MALTA DC, et al. Prevalência da hipertensão arterial segundo diferentes critérios diagnósticos, Pesquisa Nacional de Saúde. Rev. bras. epidemiol., São Paulo, 2018, 21(supl. 1): e180021.

10. MENDES G, et al. Prevalência de hipertensão arterial sistêmica em idosos no Brasil entre 2006 e 2010. RevBrasMedFam Comunidade. Rio de Janeiro. 2014. 9(32), 273-278.

11. MORAES AAL, et al. O impacto da hipertensão arterial no mundo. In: Brandão AA, et al. Hipertensão. Rio de Janeiro: Elsevier, 2012. 7(2): 11-19.

12. MOREIRA JPL, et al. A prevalência de hipertensão arterial sistêmica autorreferida nos ambientes urbanos e rural no Brasil: um estudo de base populacional. Cad Saúde Pública 2013; 29(1): 62-72.

13. NOBLAT ACB, et al. Complicações da hipertensão arterial em homens e mulheres atendidos em um ambulatório de referência. Arq Bras Cardiol 2004; 83(4): 308-13. 
14. OLIVEIRA BFA, de et al. Prevalência de hipertensão arterial em comunidades ribeirinhas do Rio Madeira, Amazônia Ocidental Brasileira. 2013. 29(8): 1617-1630.

15. PASSOS VMA, et al. Hipertensão arterial no Brasil: estimativa de prevalência a partir de estudos de base populacionalHypertension in Brazil: estimates from population-based prevalence studies. Epidemiol. Serv. Saúde, Brasília, 2006, 15(1): 35-45.

16. RADOVANOVIC CAT, et al. Hipertensão arterial e outros fatores de risco associados às doenças cardiovasculares em adultos.Revista Latino-Americana de Enfermagem, 2014 22(4): 547-553.

17. SOUSA JCL, et al. Adesão dos pacientes ao tratamento da hipertensão arterial sistêmica. R. Interd.: Centro Universitário Uninovafapi. 2014, 7(3):17-24.

18. SOUZA NP, et al. Temporal variation in prevalence, awareness and control of hypertension in urban and rural areas in Northeast Brazil between 2006 and 2016. Cad. Saúde Pública, Rio de Janeiro, 2020; 36(4): e00027819.

19. YUGAR-TOLEDO JC, et al . Posicionamento Brasileiro sobre Hipertensão Arterial Resistente - 2020. Arq. Bras. Cardiol., São Paulo, 2020, 114(3): 576-596.

20. ZATTAR LC, et al. Prevalência e fatores associados à pressão arterial elevada, seu conhecimento e tratamento em idosos no sul do Brasil Prevalence and factors associated with high blood pressure, awareness, and treatment among elderly. Cad. Saúde Pública, 2013, 29(3): 507-521. 